British Journal of Psychiatry (1995), 167, 818-826

\title{
Correspondence
}

Contents: Cognitive functioning with chronic fatigue/Comorbidity and suicide risk/Five group comparisons of treatments for anxiety disorders/ Homicidal behaviour and mental disorders/Seizure threshold in bilateral and unilateral ECT/Late-life depressive order/Keeping count for the community/ Severe apathetic hyperthyroidism with normal thyroid hormone levels/Antidepressants in pregnancy and breastfeeding/Neuropsychological function in manic-depressive psychosis/Visual fields in Gilles de la Tourette syndrome

\section{Cognitive functioning with chronic fatigue}

SIR: Cope et al (1995) claim that cognitive impairments associated with chronic fatigue "probably reflect psychopathology rather than a post-viral process". They based this conclusion on findings from a small, heterogeneous group of patients, some of whom may have been cases of true postviral fatigue syndrome, but a significant number of whom may have been suffering from previral fatigue. Given that the documented infections in the latter cases were probably coincidental, why was it assumed that their symptoms might have been caused by encephalitis? Surely, if the researchers had really wanted to assess the role of a "post-viral process" on cognitive functioning, they should have restricted their sample to patients with unambiguous post-infectious fatigue and included at least one relevant measure of immune status at the time of testing.

Although British doctors have generally been reluctant to distinguish subgroups of patients with chronic fatigue syndrome, it is noteworthy that where research has focused on more strictly defined samples, objective evidence of cognitive impairments has been found (Riccio et al, 1992; Sandman et al, 1993). Indeed, neuropsychiatric dysfunction is included as a major criterion in several case definitions of this syndrome (Lloyd et al, 1990; Lapp \& Cheney, 1995). Even the Oxford guidelines used by Cope et al require that the fatigue must affect "mental functioning".

Since the results of this study indicate that the subjective reports might have been exaggerated and even inaccurate, shouldn't the researchers have reconsidered their diagnosis of the 11 subjects with CFS? Conversely, if they took the patients' own assessments as being valid and reliable, why do they not extend this to their views of aetiology and opinions concerning exercise?

Finally, we note that Cope et al described the sample studied by Riccio et al (1992) as "depressed CFS patients". In fact, these researchers focused on myalgic encephalomyelitis (ME) and it is clear from the paper that not everyone would have fulfilled the existing diagnostic criteria for CFS. As for their psychiatric status, our copy of the article states that only $22 \%$ suffered from depression. Cope et al's rediagnosis of other researchers' patients would not have bothered us had it not preceded a jibe at experienced colleagues whose reasoned interpretation of the data they dismissed as a "misuse of neuropsychological test results".

Cope, H., Pernet, A., Kendall. B., el al (1995) Cognitive functioning and magnetic resonance imaging in chronic fatigue. British Journal of Psychiatry, 167, 86-94.

LAPP. C. W. \& CheneY, P. R. (1995) The chronic fatigue syndrome. Annals of Internal Medicine, 123, 74-75.

LLOYD, A. R., HICKIE, I., Bougrton, C. R., et al (1990) Prevalence of chronic fatigue syndrome in an Australian population. Medical Journal of Australia, 153, 522-528.

Riccio, M., Thompson, C., Wilson, B., et al (1992) Neuropsychological and psychiatric abnormalities in myalgic encephalomyelitis: a preliminary report. British Journal of Psychiatry, 31, $111-120$.

Sandman, C. A., Barron, J. L., Nackovi, K., et al (1993) Memory deficits associated with chronic fatigue immune dysfunction syndrome. Biological Psychiatry, 33, 618-623.

\section{E. M. GOUDSMIT}

S. HOWES

$M E \&$ CFS Medical Update

23 Melbourne Road

Teddington TW11 9QX

AUTHORs' REPLY: Goudsmit \& Howes take issue with the conclusions from our study, namely that subjective cognitive complaints are a prominent feature of chronic fatigue, (while objective cognitive and MRI abnormalities are not), and that such complaints probably reflect psychopathology rather than a post-viral process. 